\title{
On the Origin of the Red-Shifted Optical Spectra Recorded for aza-BODIPY Dyes
}

\section{Supporting Information}

\author{
Joshua K. G. Karlsson and Anthony Harriman* \\ Molecular Photonics Laboratory, School of Chemistry, Bedson Building, Newcastle \\ University, Newcastle upon Tyne, NE1 7RU, United Kingdom
}

\section{Table of Contents}

\section{Topic}

1. Gaussian Deconstruction of Low-Temperature Emission Spectra

2. Gaussian Deconstruction of Room-Temperature Emission Spectra

3. Gaussian Deconstruction of Absorption Spectra

4. Steady-State Photo-bleaching Experiments

5. Steady-State Anisotropy Measurements

6. Energy-minimized Structure for aza-BOD

7. Energy-minimized Structure for BOD

8. Dihedral angle computed for aza-BOD at different levels

9. Structural parameters for the excited states of BOD and aza-BOD

10. Beer's law plot for aza-BOD 
Gaussian Deconstruction of Absorption and Emission Spectra

Deconstruction of the absorption and emission spectra for aza-BOD and BOD, respectively, was accomplished by fitting each spectral trace to a minimum number of Gaussian curves of a common full-width at half-height (FWHM). It was determined that the spectra recorded for both compounds consistently fitted to the highest accuracy (measured by $\mathrm{R}^{2}$ values) as the sum of five Gaussian components. The resultant absorption and emission spectra are presented below (Figures S1-6). The y-axis is presented in units of wavenumber.

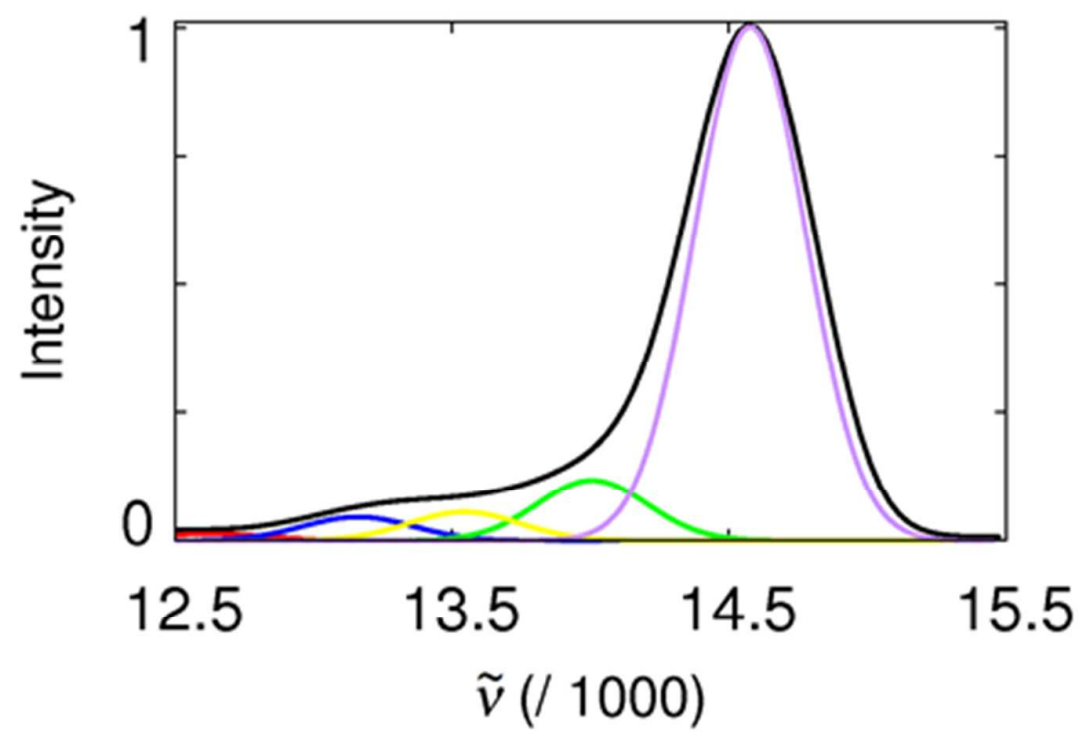

Figure S1. Low-temperature (77K) fluorescence spectrum recorded for aza-BOD.

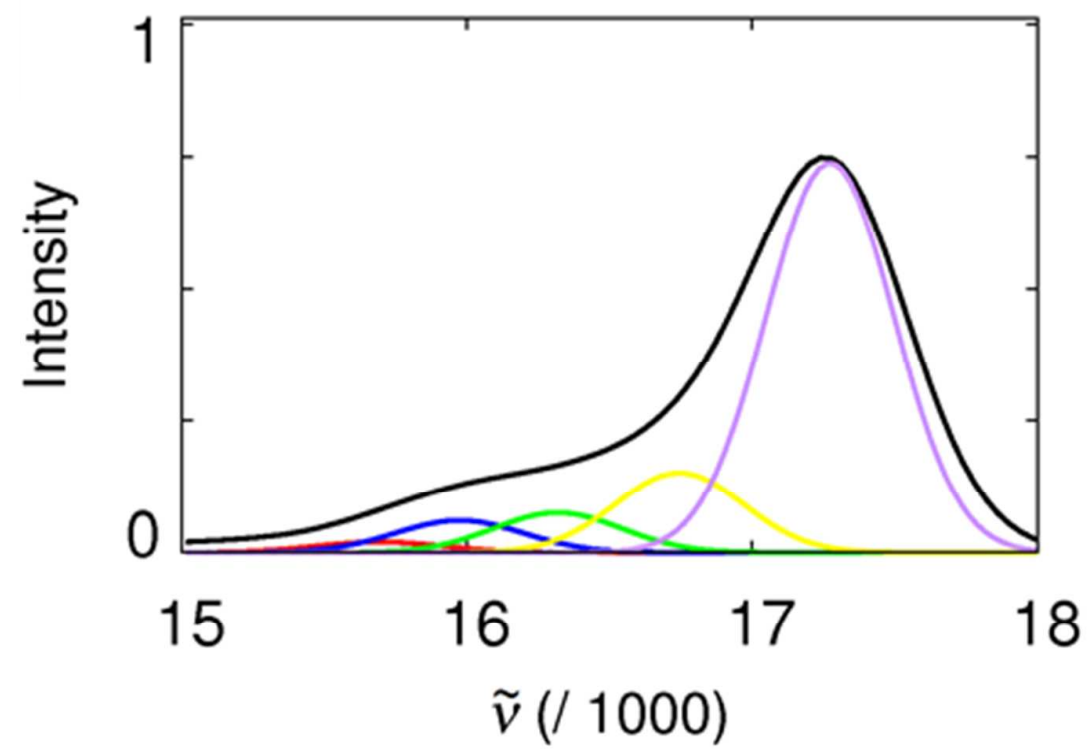

Figure S2. Low temperature (77K) fluorescence spectrum recorded for BOD. 


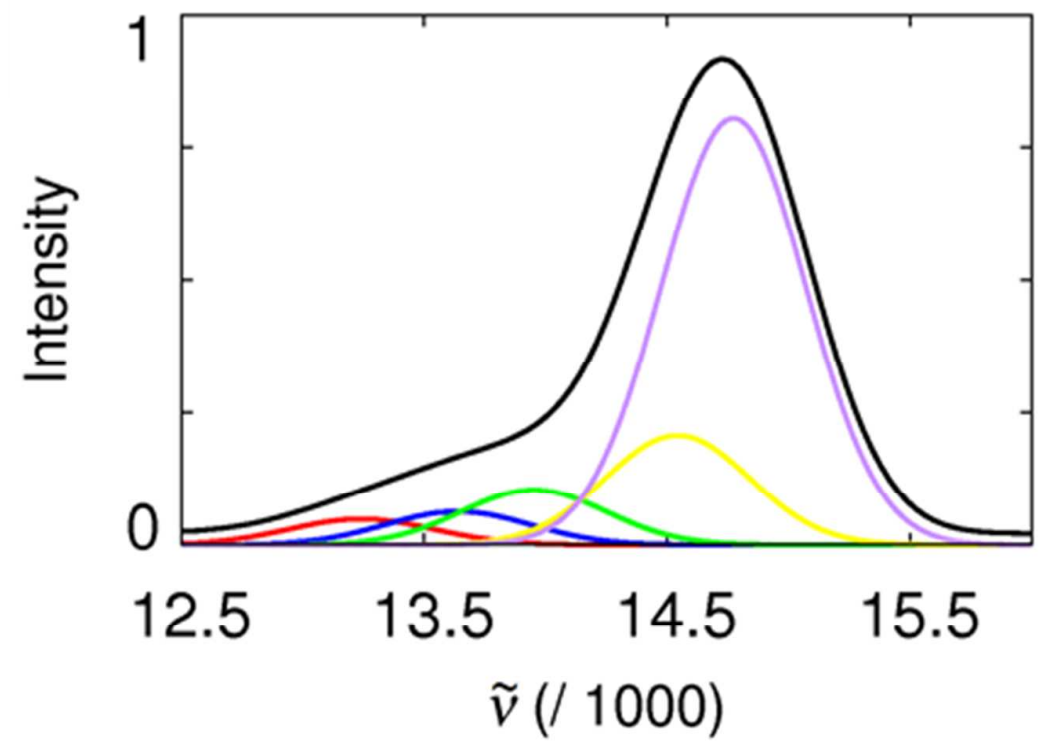

Figure S3. Room-temperature fluorescence spectrum recorded for aza-BOD.

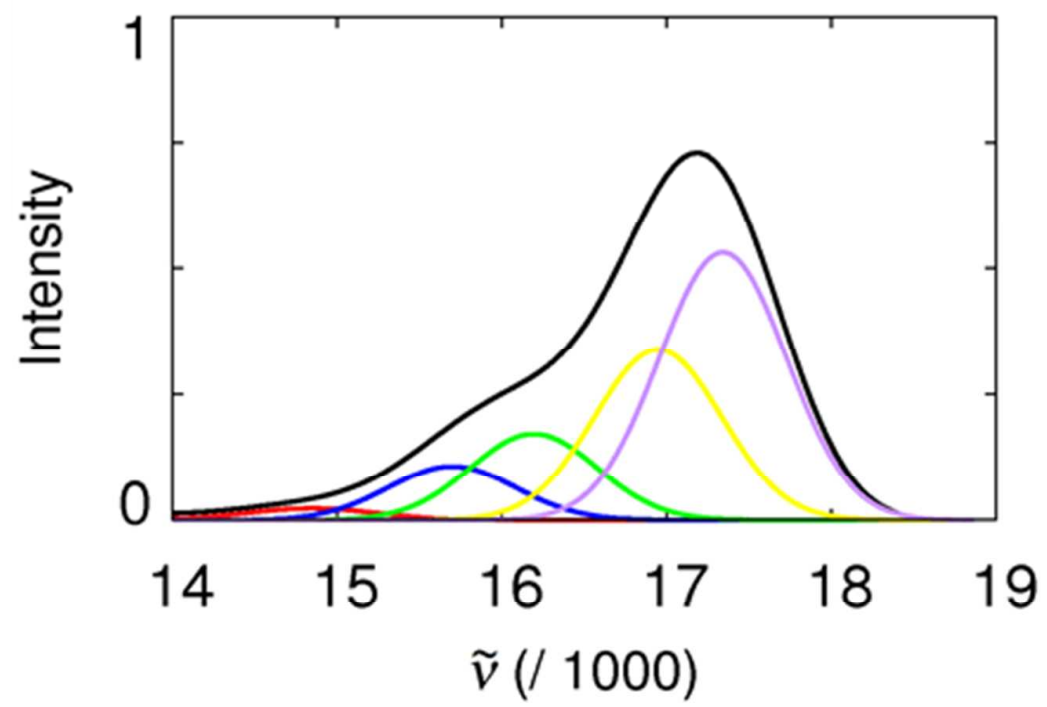

Figure S4. Room-temperature fluorescence spectrum recorded for BOD. 


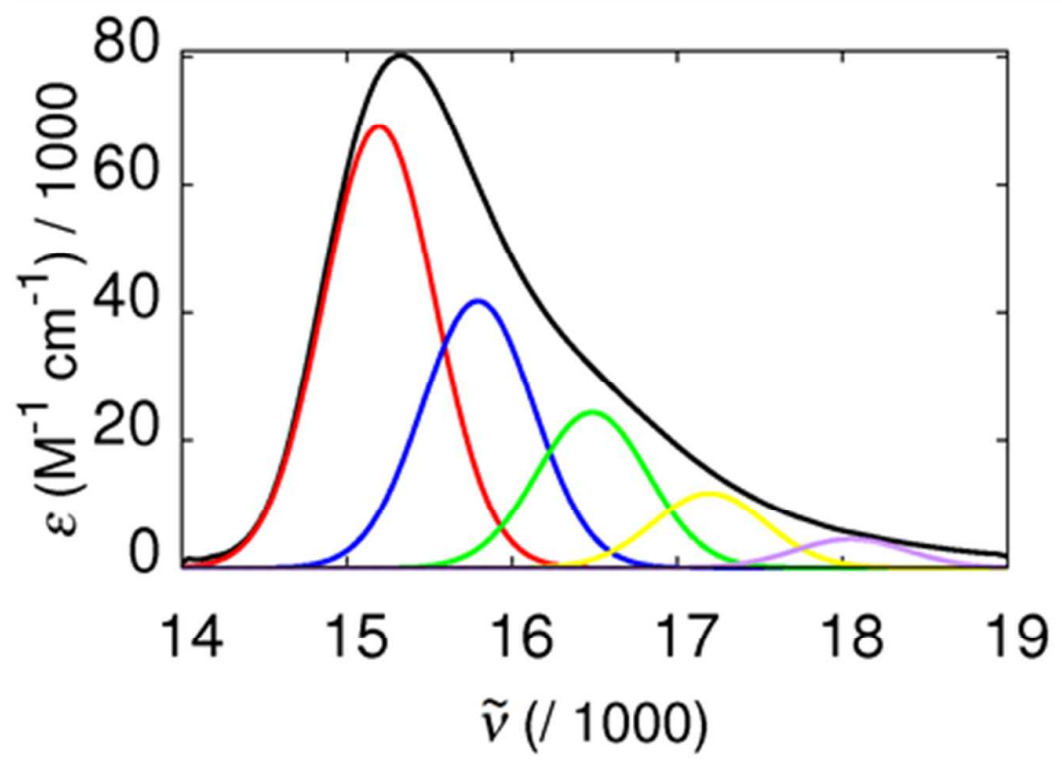

Figure S5. Absorption spectrum recorded for aza-BOD.

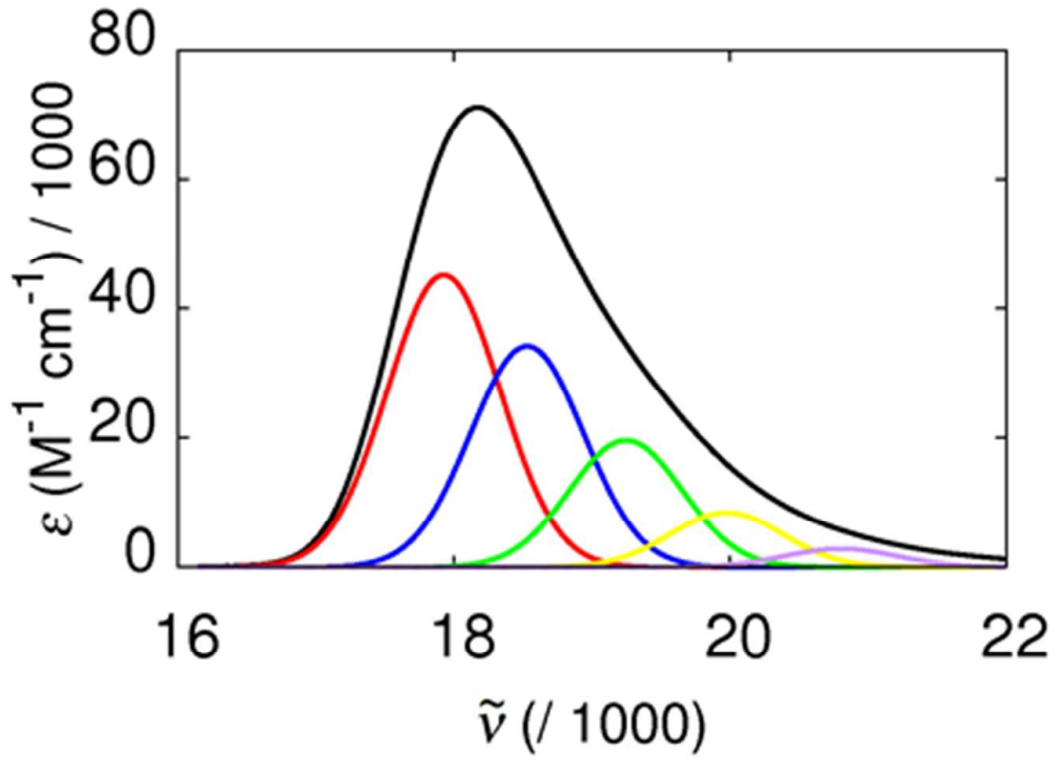

Figure S6. Absorption spectrum recorded for BOD. 


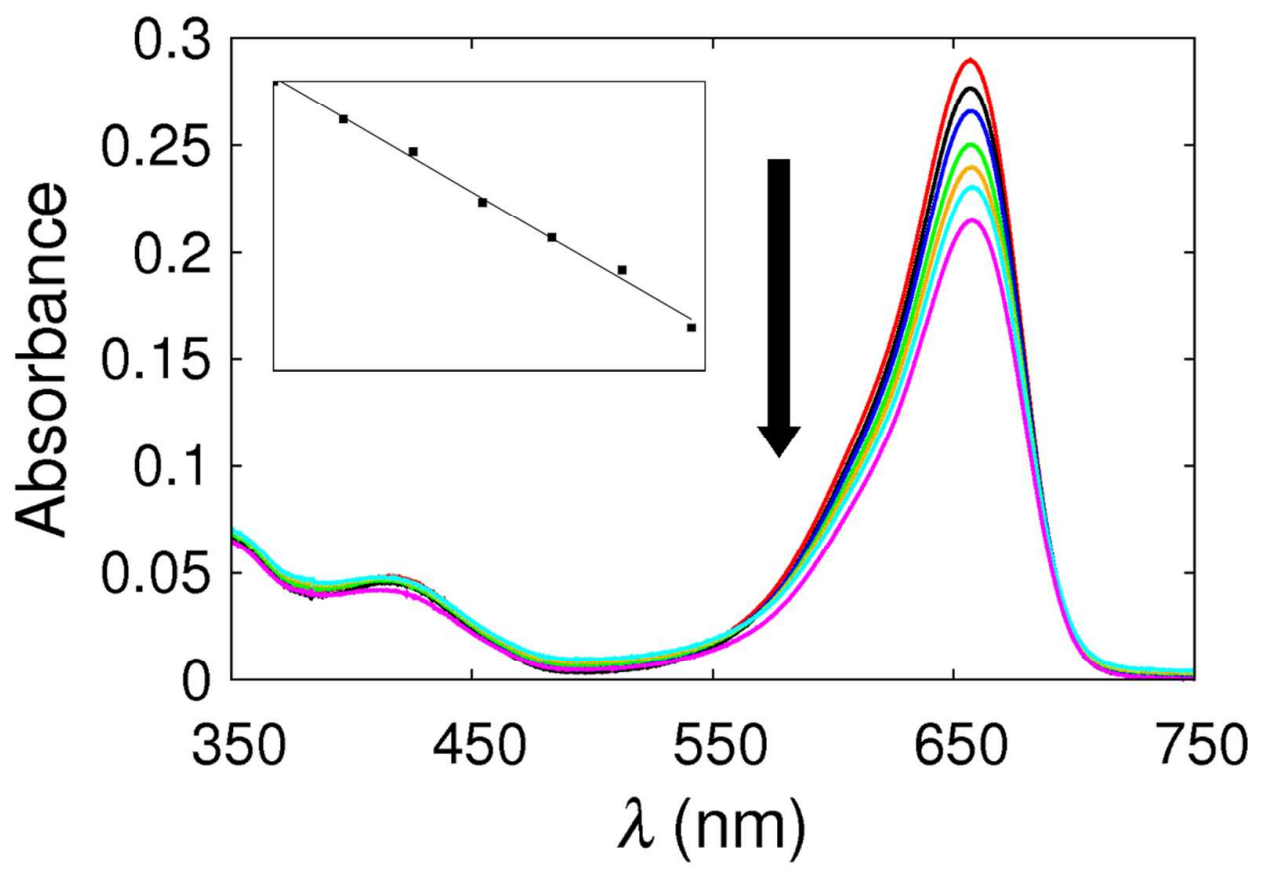

Figure S7. Steady-state photo-bleaching of aza-BOD in deaerated DMF. Absorption spectra recorded at equal periods between 0 and 6 hours. A semi-log plot (inset) of absorbance as a function of time $(\mathrm{x})$ reveals bleaching consistent with first-order kinetics. On this basis, the rate constant for photo-bleaching was determined as $6.0 \times 10^{-7} \mathrm{M} \mathrm{h}^{-1}$ (error limits $\pm 10 \%$ ).

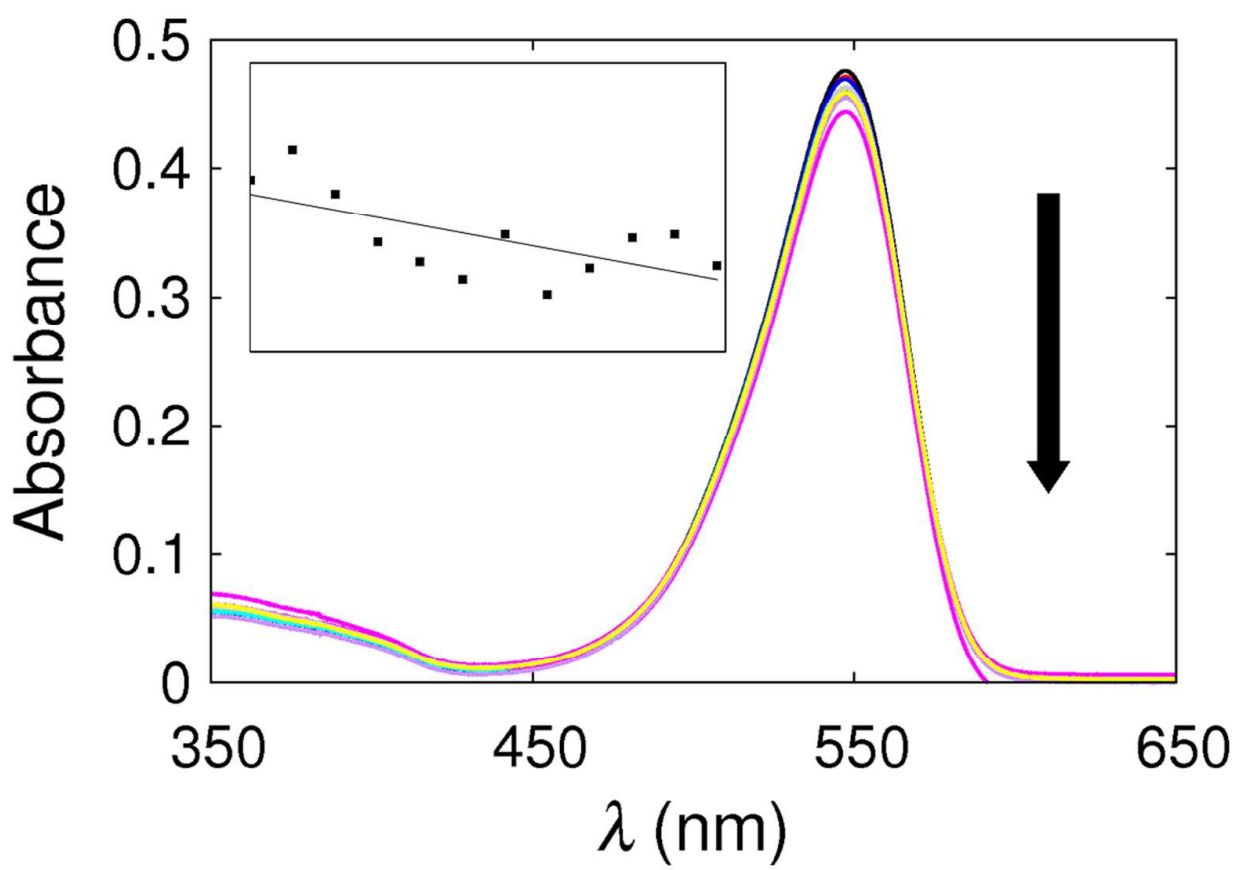

Figure S8. Steady-state photo-bleaching of BOD in deaerated DMF. Absorption spectra recorded at equal intervals between 0 and 10 hours. A semi-log plot of absorbance values as a function of time is given as the inset. Negligible bleaching was observed in comparison with aza-BOD. 


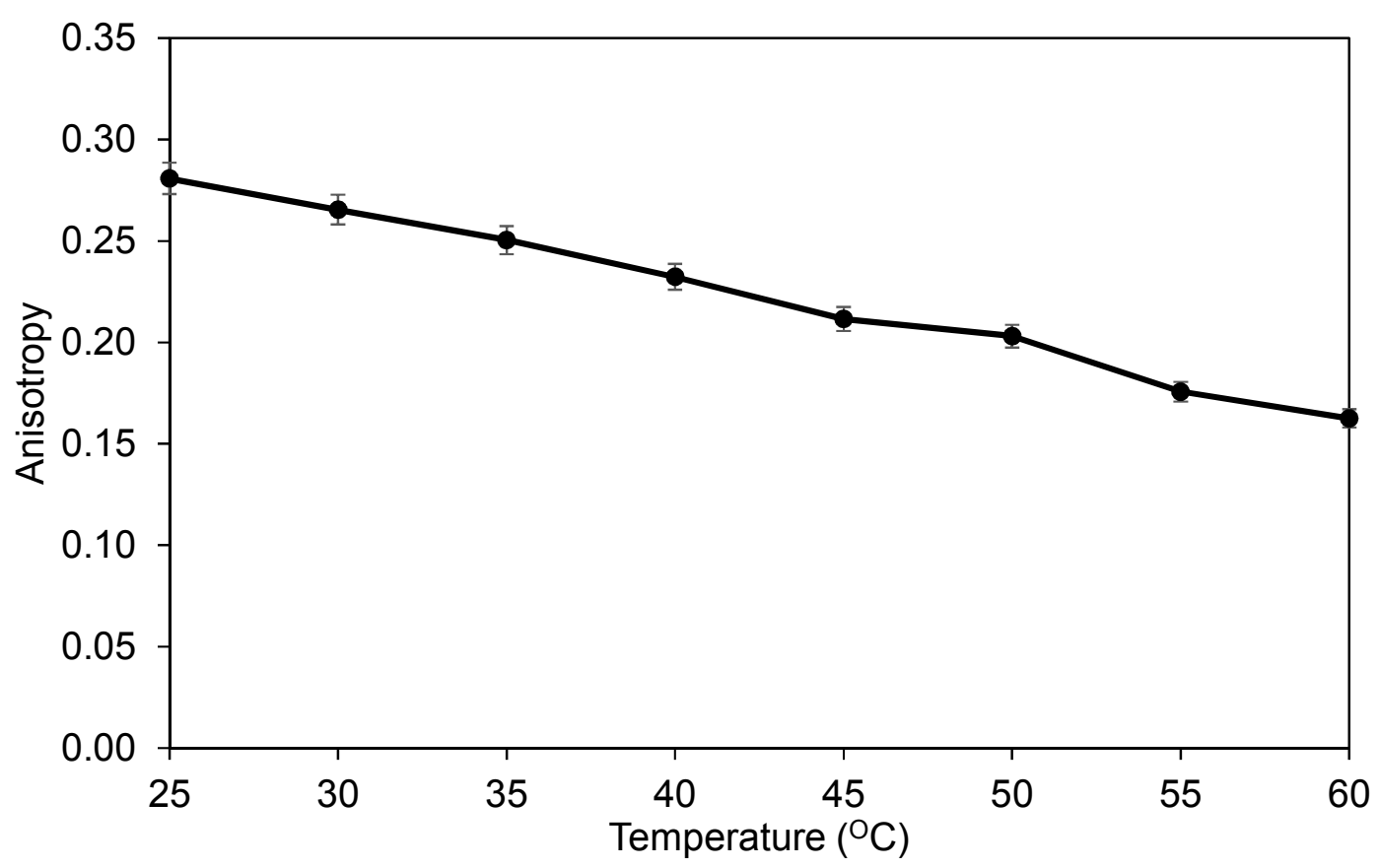

Figure S9. Steady-state fluorescence anisotropy measurements for aza-BOD in glycerol as a function of temperature. Measurements were repeated 8 times with an average experimental error of $2.8 \%$. Modelling suggests the limiting anisotropy value for aza-BOD is 0.37 , which is consistent with literature values for somewhat related compounds.

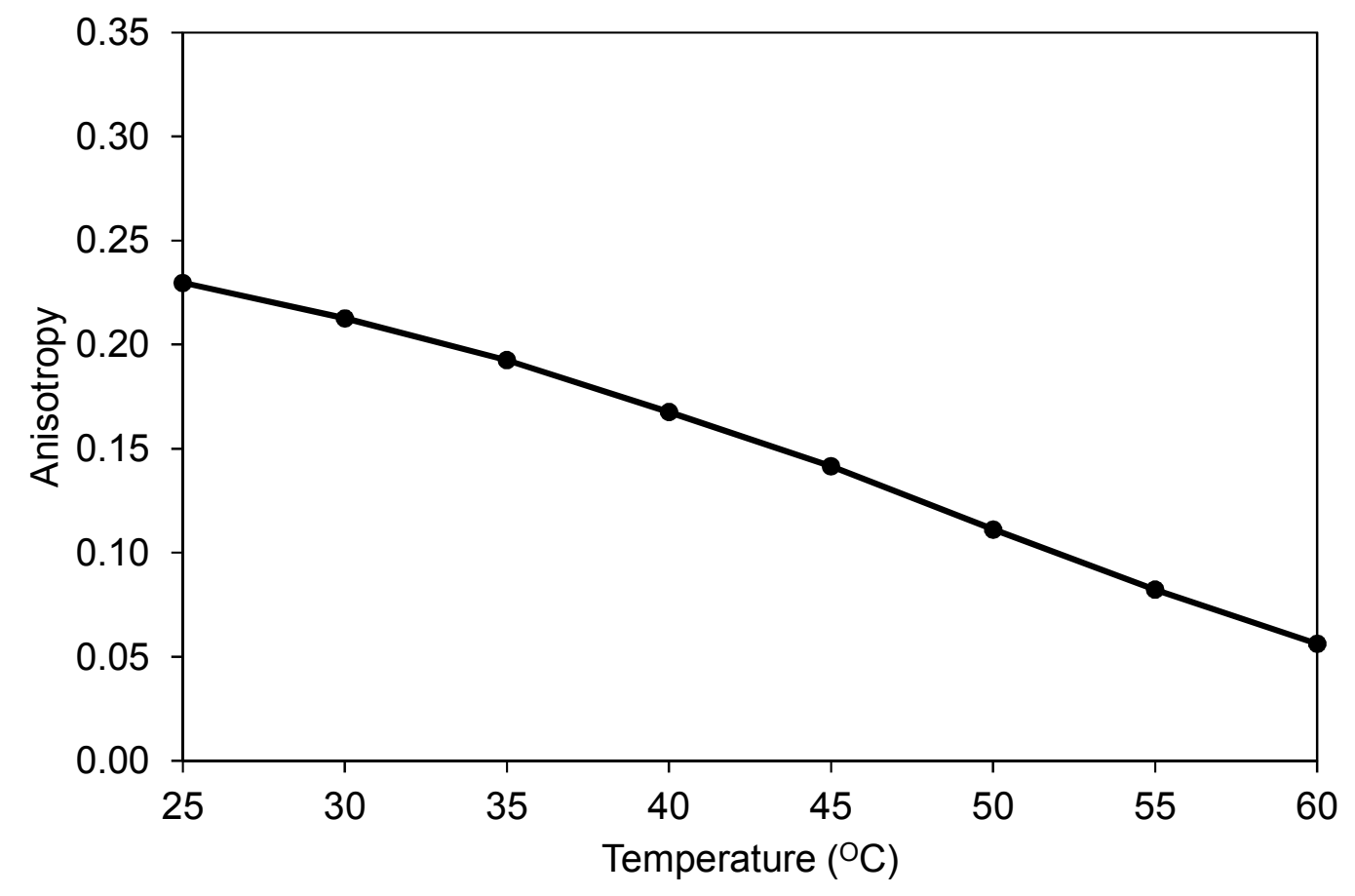

Figure S10. Steady-state fluorescence anisotropy measurements for BOD in glycerol as a function of temperature. Measurements were repeated 8 times with an average experimental error of $0.9 \%$. 
Energy-minimized geometry for aza-BOD

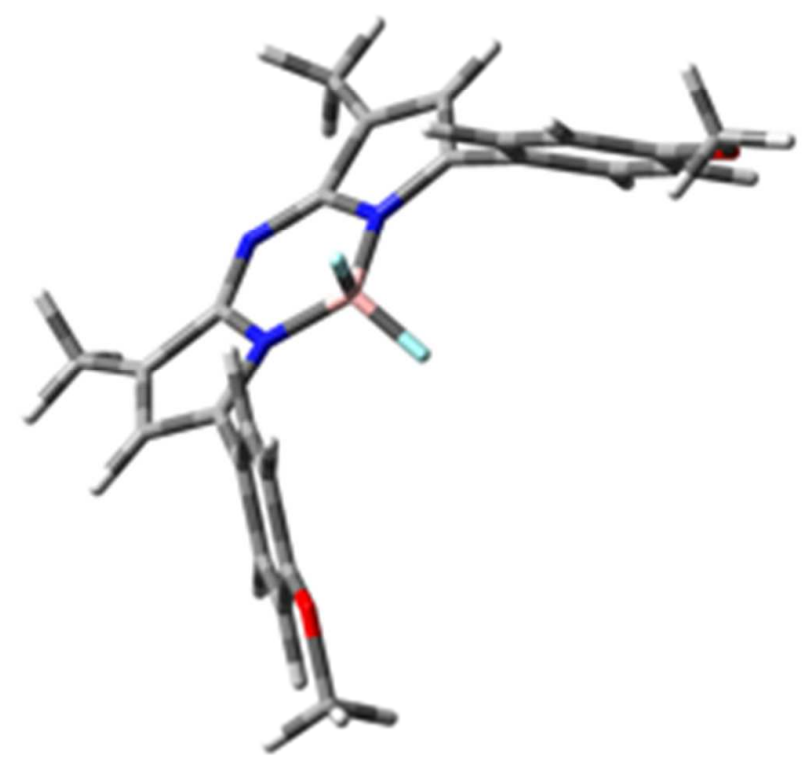

CARTESIAN COORDINATES

$\begin{array}{cccc}\text { NO. } & \text { ATOM } & \text { X } & Y \\ \text { 1C } & 0.00000000 & 0.00000000 & 0.00000000 \\ \text { 2 N } & 1.45220000 & 0.00000000 & 0.00000000 \\ \text { 3 B } & 2.26079986 & 1.34519977 & 0.00000000 \\ \text { 4 N } & 1.24199963 & 2.53860005 & -0.04560001 \\ \text { 5 C } & -0.19070019 & 2.30000008 & -0.04570001 \\ \text { 6 N } & -0.75770032 & 1.09559983 & -0.02010000 \\ \text { 7 C } & 1.45769962 & 3.87080010 & -0.11630001 \\ \text { 8C } & 0.18209921 & 4.55640031 & -0.16160003 \\ \text { 9 C } & -0.82960035 & 3.61580042 & -0.11290003 \\ \text { 10 C } & -0.41459975 & -1.40350057 & -0.01430000 \\ \text { 11 C } & 0.73780017 & -2.16700005 & -0.03600001 \\ \text { 12 C } & 1.88350012 & -1.28080036 & -0.02540000 \\ \text { 13 C } & -2.28230021 & 3.82550041 & -0.12500003 \\ \text { 14 C } & -1.81309968 & -1.84940096 & -0.00340000 \\ \text { 15 C } & 3.28340058 & -1.70580050 & -0.05110001 \\ \text { 16 C } & 2.77000002 & 4.51710031 & -0.15030001 \\ \text { 17 F } & 3.12690010 & 1.39829980 & -1.06850030 \\ \text { 18 F } & 3.06259965 & 1.43259975 & 1.11639968 \\ \text { 19 C } & 3.67981297 & 4.34973412 & 0.90397380 \\ \text { 20 C } & 4.92310611 & 4.98392189 & 0.87216871 \\ \text { 21 C } & 5.24119826 & 5.78121055 & -0.23481297 \\ \text { 22 C } & 4.33734588 & 5.96579194 & -1.30425160 \\ \text { 23 C } & 3.10506160 & 5.33030217 & -1.25257292 \\ \text { 24 C } & 4.16137441 & -1.35056715 & 0.99221348 \\ \text { 25 C } & 5.48370519 & -1.77288455 & 0.96672889 \\ \text { 26 C } & 5.92760246 & -2.55239960 & -0.12301259\end{array}$




$\begin{array}{llll}27 \mathrm{C} & 5.06874610 & -2.91834835 & -1.16841057 \\ 28 \mathrm{C} & 3.74207559 & -2.48893117 & -1.12193504 \\ 29 \mathrm{O} & 7.25399173 & -2.89375048 & -0.02824146 \\ 30 \mathrm{C} & 7.83119997 & -3.70861047 & -1.08309041 \\ 31 \mathrm{O} & 6.41968897 & 6.46295846 & -0.41123295 \\ 32 \mathrm{C} & 7.44298288 & 6.34260090 & 0.61271864 \\ 33 \mathrm{H} & 0.08059923 & 5.62500007 & -0.21900001 \\ 34 \mathrm{H} & 0.81230046 & -3.23920005 & -0.05340001 \\ 35 \mathrm{H} & -2.56190033 & 4.88910089 & -0.17740006 \\ 36 \mathrm{H} & -2.75980040 & 3.31920022 & -0.98310035 \\ 37 \mathrm{H} & -2.76230002 & 3.40510060 & 0.77689959 \\ 38 \mathrm{H} & -2.39890001 & -1.38360095 & -0.81560028 \\ 39 \mathrm{H} & -1.91619933 & -2.94040054 & -0.10909995 \\ 40 \mathrm{H} & -2.32109993 & -1.56410100 & 0.93560032 \\ 41 \mathrm{H} & 3.41518203 & 3.72798635 & 1.76341644 \\ 42 \mathrm{H} & 5.62082536 & 4.84858913 & 1.69552809 \\ 43 \mathrm{H} & 4.61846161 & 6.59584567 & -2.14682211 \\ 44 \mathrm{H} & 2.39110646 & 5.45649015 & -2.07063622 \\ 45 \mathrm{H} & 3.79855980 & -0.75070951 & 1.83093006 \\ 46 \mathrm{H} & 6.17866340 & -1.51266082 & 1.76384664 \\ 47 \mathrm{H} & 5.41484213 & -3.52101051 & -2.00492142 \\ 48 \mathrm{H} & 3.05756671 & -2.76323105 & -1.92893681 \\ 49 \mathrm{H} & 8.86786062 & -3.82557937 & -0.73856468 \\ 50 \mathrm{H} & 7.32497077 & -4.67699703 & -1.13643639 \\ 51 \mathrm{H} & 7.79386307 & -3.17520755 & -2.03781257 \\ 52 \mathrm{H} & 8.24939549 & 6.96596870 & 0.20274109 \\ 53 \mathrm{H} & 7.07909205 & 6.74720160 & 1.56187928 \\ 54 \mathrm{H} & 7.76412045 & 5.30111394 & 0.70930467\end{array}$


Energy-

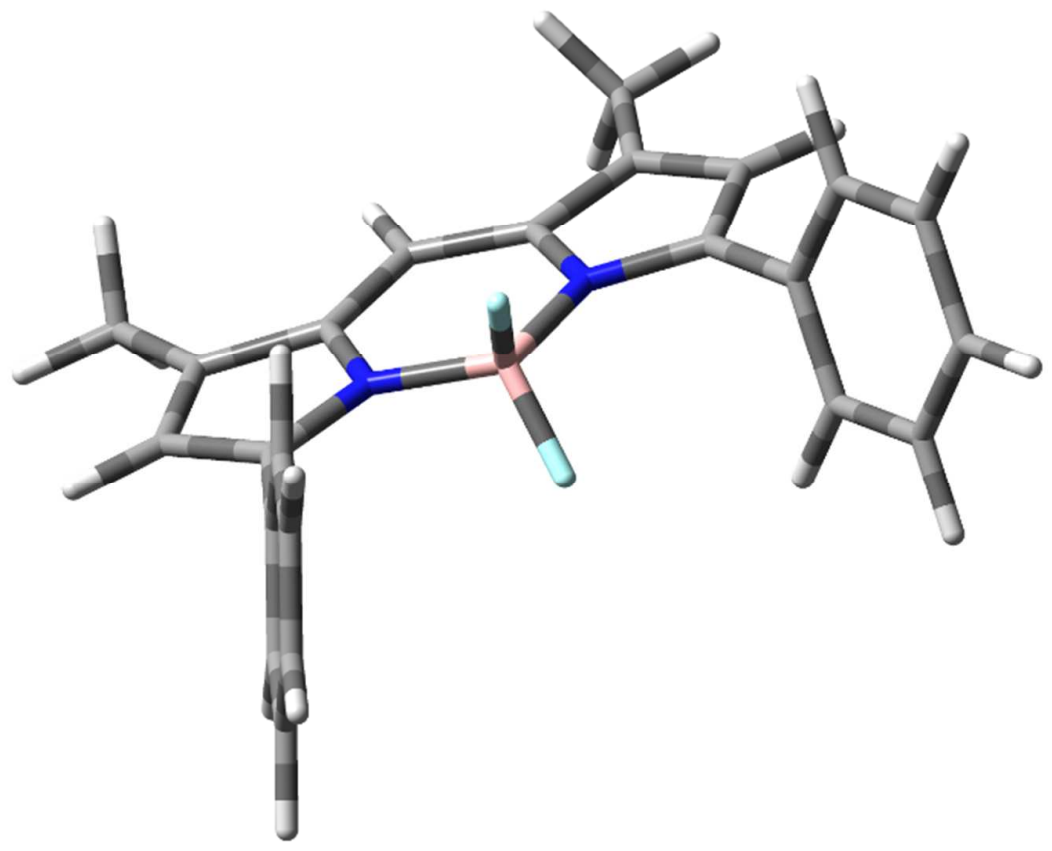

$\underline{\text { minimized geometry for BOD }}$

\section{CARTESIAN COORDINATES}

NO. ATOM $X \quad Y \quad Z$

$\begin{array}{llll}1 C & 0.00000000 & 0.00000000 & 0.00000000\end{array}$ 


\begin{tabular}{|c|c|c|c|}
\hline $2 \mathrm{~N}$ & 1.43140000 & 0.00000000 & 0.00000000 \\
\hline $3 \mathrm{~B}$ & 2.26279983 & 1.32499971 & 0.00000000 \\
\hline$N$ & 1.30610003 & 2.55979948 & 0.07910000 \\
\hline C & -0.11830042 & 2.42199946 & 0.06860000 \\
\hline $6 \mathrm{C}$ & -0.73570025 & 1.17810041 & 0.02700002 \\
\hline $7 \mathrm{C}$ & 1.61480004 & 3.87779950 & 0.14510001 \\
\hline $8 \mathrm{C}$ & 0.39820040 & 4.64709927 & 0.17940002 \\
\hline $9 \mathrm{C}$ & -0.67089946 & 3.76069938 & 0.12970003 \\
\hline $10 \mathrm{C}$ & -0.42090014 & -1.38740045 & -0.00630001 \\
\hline C & 0.72869950 & -2.16770019 & -0.00110000 \\
\hline $2 \mathrm{C}$ & 1.86569990 & -1.28379969 & 0.00120000 \\
\hline $13 \mathrm{C}$ & -2.10809906 & 4.07449928 & 0.13440005 \\
\hline $14 \mathrm{C}$ & -1.82120058 & -1.83810059 & -0.01260001 \\
\hline $5 \mathrm{C}$ & 3.27569957 & -1.69809957 & 0.00950000 \\
\hline $6 \mathrm{C}$ & 2.97940009 & 4.42259952 & 0.18350002 \\
\hline $7 \mathrm{~F}$ & 3.04729959 & 1.40049968 & -1.13279964 \\
\hline $18 \mathrm{~F}$ & 3.15729997 & 1.33569970 & 1.04970015 \\
\hline $19 \mathrm{C}$ & 3.78474445 & 4.34512898 & -0.96337926 \\
\hline $20 \mathrm{C}$ & 5.09142216 & 4.83678249 & -0.92391670 \\
\hline $21 \mathrm{C}$ & 5.59456679 & 5.40165029 & 0.25285507 \\
\hline $22 \mathrm{C}$ & 4.79006982 & 5.47734137 & 1.39384435 \\
\hline $23 \mathrm{C}$ & 3.48037818 & 4.99004965 & 1.36392173 \\
\hline $24 \mathrm{C}$ & 4.04631583 & -1.49182069 & 1.16431475 \\
\hline $25 \mathrm{C}$ & 5.37912856 & -1.90867538 & 1.18913562 \\
\hline $26 \mathrm{C}$ & 5.94165061 & -2.53090812 & 0.06957442 \\
\hline $27 \mathrm{C}$ & 5.17148242 & -2.73611018 & -1.07909761 \\
\hline $28 \mathrm{C}$ & 3.83746475 & -2.32091648 & -1.11452215 \\
\hline $29 \mathrm{H}$ & -1.83369987 & 1.12470043 & 0.02240002 \\
\hline $30 \mathrm{H}$ & 0.35930039 & 5.71809947 & 0.23270003 \\
\hline $31 \mathrm{H}$ & 0.79369947 & -3.23879971 & -0.00090001 \\
\hline $32 \mathrm{H}$ & -2.30489902 & 5.15439894 & 0.22840002 \\
\hline $33 \mathrm{H}$ & -2.60319887 & 3.74159942 & -0.79369957 \\
\hline $34 \mathrm{H}$ & -2.63659899 & 3.58159934 & 0.96729996 \\
\hline $35 \mathrm{H}$ & -2.38060040 & -1.43730072 & -0.87429971 \\
\hline $36 \mathrm{H}$ & -1.91210059 & -2.93510065 & -0.05740002 \\
\hline $37 \mathrm{H}$ & -2.36110057 & -1.51220060 & 0.89269999 \\
\hline $38 \mathrm{H}$ & 3.38927629 & 3.90806857 & -1.88080787 \\
\hline $39 \mathrm{H}$ & 5.71935209 & 4.78070925 & -1.81372497 \\
\hline $40 \mathrm{H}$ & 6.61532030 & 5.78454880 & 0.27960224 \\
\hline $41 \mathrm{H}$ & 5.18271202 & 5.91955222 & 2.31003326 \\
\hline $42 \mathrm{H}$ & 2.85089893 & 5.05385004 & 2.25234049 \\
\hline $43 \mathrm{H}$ & 3.60672806 & -1.00422052 & 2.03510101 \\
\hline $44 \mathrm{H}$ & 5.98152138 & -1.74742936 & 2.08369136 \\
\hline $45 \mathrm{H}$ & 6.98265643 & -2.85529631 & 0.09295080 \\
\hline $46 \mathrm{H}$ & 5.61147405 & -3.21998751 & -1.95172706 \\
\hline $47 \mathrm{H}$ & 3.23755786 & -2.47943864 & -2.01151725 \\
\hline
\end{tabular}


Table S1. The Aryl Ring Dihedral Angle Calculated for aza-BOD in a Solvent Reservoir from Optimized Geometries Obtained at a Few Different Levels

\begin{tabular}{|c|c|}
\hline Method & N-C-aryl $/{ }^{0}$ \\
\hline HF/6-31G** & 54.1 \\
\hline MP2/6-31G** & 53.2 \\
\hline CCD/6-31G** & 53.5 \\
\hline B3LYP/cc-pVDZ & 54.2 \\
\hline B3LYP/cc-pVTZ & 54.3 \\
\hline B3LYP/6-31G** & 54.2 \\
\hline
\end{tabular}

Table S2. Compilation of the Structural Parameters Calculated for the Two Dyes at the Ground State and Excited State Levels. ${ }^{(a)}$

\begin{tabular}{|c|c|c|c|c|c|c|}
\hline Parameter & $\begin{array}{c}\text { BOD } \\
\left(\mathrm{S}_{0}\right)\end{array}$ & $\begin{array}{c}\text { BOD } \\
\left(\mathrm{S}_{1}\right)\end{array}$ & $\begin{array}{c}\text { BOD } \\
\left(\mathrm{T}_{1}\right)\end{array}$ & $\begin{array}{c}\text { aza-BOD } \\
\left(\mathrm{S}_{0}\right)\end{array}$ & $\begin{array}{c}\text { aza-BOD } \\
\left(\mathrm{S}_{1}\right)\end{array}$ & $\begin{array}{c}\text { aza-BOD } \\
\left(\mathrm{T}_{1}\right)\end{array}$ \\
\hline $\mathrm{C}-\mathrm{X}-\mathrm{C} /{ }^{0}$ & 121.6 & 121.7 & 119.2 & 116.2 & 119.6 & 116.8 \\
\hline $\mathrm{N}-\mathrm{B}-\mathrm{N} /{ }^{0}$ & 110.1 & 109.1 & 107.7 & 108.3 & 107.3 & 106.0 \\
\hline $\mathrm{F}-\mathrm{B}-\mathrm{F} /{ }^{0}$ & 104.8 & 106.8 & 106.7 & 105.3 & 107.1 & 107.2 \\
\hline $\mathrm{N}-\mathrm{C}-\mathrm{Ph} /{ }^{0}$ & 59.3 & 55.4 & 60.2 & 54.1 & 52.0 & 57.3 \\
\hline $\mathrm{C}-\mathrm{X} / \AA$ & 1.398 & 1.389 & 1.403 & 1.332 & 1.330 & 1.364 \\
\hline $\mathrm{N}-\mathrm{B} / \AA$ & 1.564 & 1.572 & 1.573 & 1.570 & 1.575 & 1.578 \\
\hline $\mathrm{B}-\mathrm{F} / \AA$ & 1.380 & 1.373 & 1.372 & 1.377 & 1.370 & 1.369 \\
\hline $\mathrm{X}-\mathrm{B} / \AA$ & 3.002 & 3.034 & 3.034 & 3.030 & 3.045 & 3.077 \\
\hline $\mathrm{C}_{1}-\mathrm{C}_{7} / \AA$ & 5.921 & 5.932 & 5.930 & 5.700 & 5.701 & 5.697 \\
\hline
\end{tabular}

(a) $\mathrm{S}_{0}$ refers to the ground state; $\mathrm{S}_{1}$ refers to the first-excited singlet state; $\mathrm{T}_{1}$ refers to the first-allowed triplet state. Calculations made in a solvent reservoir with dielectric constant of 10 . 


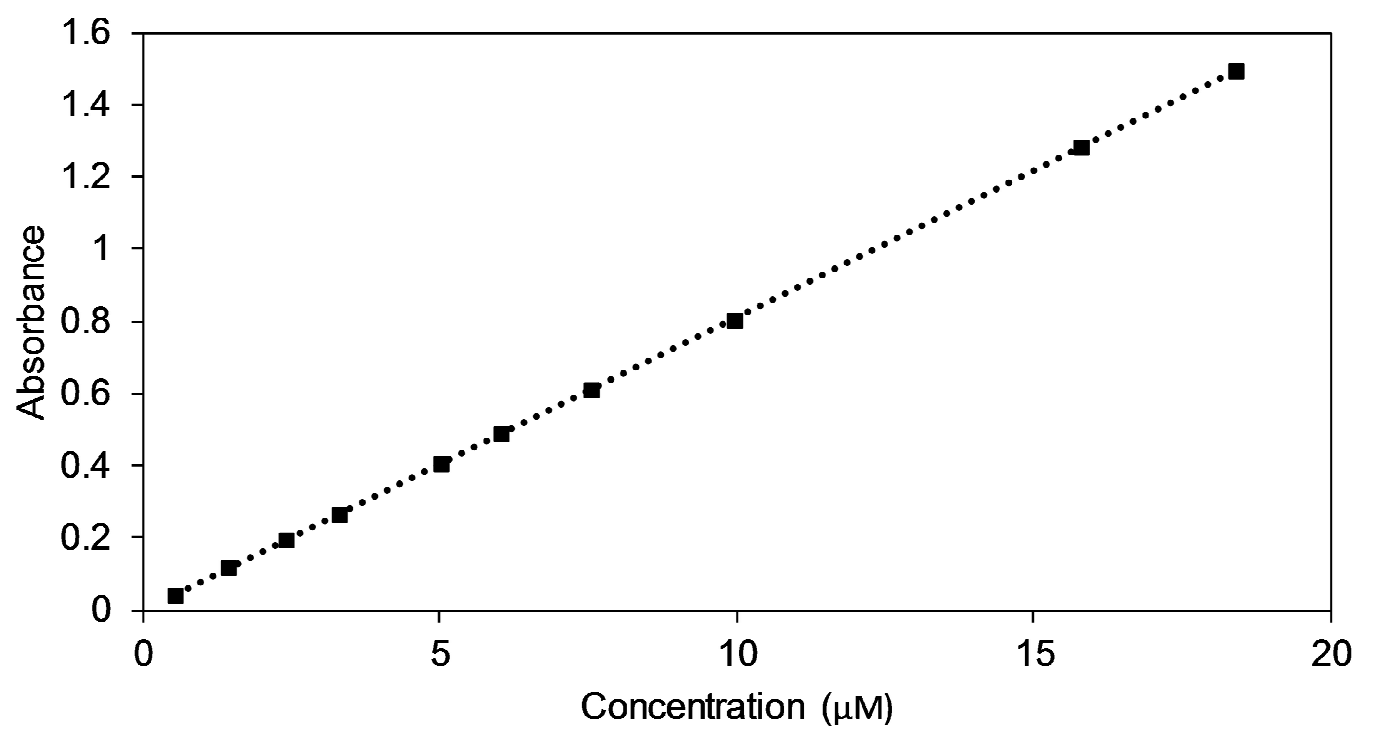

Figure S11. Example of a Beer's law plot made for aza-BOD in N,N-dimethylformamide at room temperature. 